\title{
STANDARD GRADIENT MODELS AND CRACK SIMULATION
}

\author{
Nguyen Quoc Son, Nguyen Truong Giang \\ Laboratoire de Mecanique des Solides \\ Ecole Polytechnique, 91128 Palaiseau, France
}

\begin{abstract}
The standard gradient models have been intensively studied in the literature, cf. Fremond (1985) or Gurtin (1991) for various applications in plasticity, damage mechanics and phase change analysis. The governing equations for a solid have been introduced essentially from an extended version of the virtual equation. It is shown here first that these equations can also be derived from the formalism of energy and dissipation potentials and appear as a generalized Biot equation for the solid. In this spirit, the governing equations for higher gradient models can be straightforwardly given. The interest of gradient models is then discussed in the context of damage mechanics and crack simulation. The phenomenon of strain localization in a time-dependent or timeindependent process of damage is explored as a convenient numerical method to simulate the propagation of cracks, in relation with some recent works of the literature, cf. Bourdin \& Marigo [3], Lorentz \& al [5], Henry \& al [12].
\end{abstract}

Keywords: Gradient and higher gradient models, damage mechanics, standard viscoplasticity, localization and crack propagation.

\section{INTRODUCTION}

The introduction of the gradients of the state variables such as the strain, the internal parameter and even the temperature in Solid Mechanics has been much discussed in the literature since the pioneering works of Mindlin and Toupin in second-gradient elasticity. Especially, in the two last decades, standard gradient theories has been considered in many papers, cf. for example [4], [6], [8] , [9], [18], for the modeling of phase change and of solids with microstructures. In particular, in Frémond or Gurtin's approach, the governing equations have been originally derived from an additional virtual work equation. These models have been applied in various applications such as gradient plasticity and gradient damage.

The objective of this paper is to revisit the proposed approach. Using the formalism of the generalized standard materials [16], it is first shown that a dissipation analysis can be considered in order to derive the general expression of the governing equations for the internal parameters in terms of the energy and dissipation potentials. Gradient and higher-gradient models can be discussed in the same spirit. In particular, the governing 
equations for the standard gradient models can be written as a generalized Biot equation for the solid.

The interest of gradien models is then discussed in the context of damage mechanics and crack simulation as an illustrating example. The phenomenon of strain localization in a time-dependent or time-independent process of damage is explored as a convenient numerical method to simulate the propagation of cracks, in the light of some recent works of the literature, cf. Bourdin \& Marigo [3], Lorentz \& al [5], Henry \& al [12].

\section{STANDARD GRADIENT MODELS}

In the internal variable framework, the thermo-mechanical response of a solid $V$ in a reference configuration is described by the fields of displacement $u$, of internal parameter $\phi$ and of temperature $T$. The internal parameter is a scalar or a tensor and represent physically hidden parameters such as micro-displacements or phase proportions or anelastic strains, etc.

Standard gradient models for the internal parameter assume that the set of state variables $(\nabla u, \phi, \nabla \phi, T)$ is necessary and sufficient to describe the material behaviour. The constitutive equations can be given in the following way (cf. Fremond , Gurtin,..) in an isothermal transformation:

\subsection{Generalized Forces and Virtual Work Equation}

It is first accepted that the state variables $(\nabla u, \phi, \nabla \phi)$ are associated with the generalized forces $\sigma, X, Y$ such that a generalized virtual work equation holds:

$$
P_{i}+P_{j}=P_{e} \quad \forall \delta u, \delta \phi
$$

with

$$
\begin{aligned}
& P_{i}=\int_{V}(\sigma \cdot \nabla \delta u+X \cdot \delta \phi+Y \cdot \nabla \delta \phi) d V \\
& P_{j}=\int_{V} \rho \ddot{u} \cdot \delta u d V \\
& P_{e}=\int_{V}\left(f_{v u} \cdot \delta u+f_{v \phi} \cdot \delta \phi\right) d V+\int_{\partial V}\left(f_{s u} \cdot \delta u+f_{s \phi} \cdot \delta \phi\right) d a
\end{aligned}
$$

where $\left(f_{v u}, f_{s u}\right)$ and $\left(f_{v \phi}, f_{s \phi}\right)$ are respectively external body and surface forces associated with the dispacement and the internal parameter. This means that the mechanical equilibrium equations hold for the stress

$$
\begin{array}{ll}
\nabla \cdot \sigma+f_{v u}=\rho \ddot{u} & \forall x \in V \\
\sigma \cdot n=f_{s u} & \forall x \in \partial V
\end{array}
$$

and the following constitutive equilibrium equations hold for the internal parameter after intergration by parts

$$
\begin{array}{ll}
\nabla \cdot Y-X+f_{v \phi}=0 & \forall x \in V \\
Y \cdot n=f_{s \phi} & \forall x \in \partial V
\end{array}
$$

These equations are easily understood when $\phi$ is a micro-displacement, $X$ is then an internal volume force and $Y$ is a micro-stress in the same spirit as stress $\sigma$. 


\subsection{Energy and Dissipation Potentials}

Standard gradient models also assume that there exist an energy potential $w$ and a dissipation potential $D$ per unit reference volume such that the following equations hold:

$$
\begin{aligned}
& w=w(\nabla u, \phi, \nabla \phi), \quad D=D(\nabla \dot{u}, \dot{\phi}, \nabla \dot{\phi}, \nabla u, \phi) \\
& \sigma=\sigma_{e}+\sigma_{d}, \quad \sigma_{e}=w, \nabla u, \quad \sigma_{d}=D, \nabla \dot{u} \\
& X=X_{e}+X_{d}, \quad X_{e}=w, \phi, \quad X_{d}=D, \dot{\phi} \\
& Y=Y_{e}+Y_{d}, \quad Y_{e}=w, \nabla \phi, \quad Y_{d}=D, \nabla \dot{\phi}
\end{aligned}
$$

when the potentials $w$ and $D$ are respectively smooth functions with respect to the state variables and the fluxes and the dissipation potential is assumed to be state-dependent via the current value of $\nabla u, \phi$. The force-flux relationships $X_{d}=D, \dot{\phi}, Y_{d}=D, \nabla \dot{\phi}$ describe a time-dependent behaviour of the materials and are commonly discussed in Visco-Elasticity, Visco-plasticity, in Phase change as in Damage Mechanics.

The case of convex but non-smooth dissipation potentials is also interesting in Solids Mechanics. For example, $D$ is a convex, positive homogeneous of degree 1 in timeindependent processes such as friction, plasticity, brittle fracture and brittle damage. In this case, the relationships between dissipative forces and fluxes in (5) must be specified as in Classical Plasticity, cf. [15].

\subsection{Governing Equations}

The equations (3), (4),(5) are the governing equations of a standard gradient model. In terms of the two potentials, the governing equations for the fields of unknown $\mathbf{u}, \boldsymbol{\Phi}$ are

$$
\begin{array}{ll}
\nabla \cdot(w, \nabla u+D, \nabla \dot{u})+f_{v u}=\rho \ddot{u} & \forall x \in V \\
(w, \nabla u+D, \nabla \dot{u}) \cdot n=f_{s u} & \forall x \in \partial V \\
\nabla \cdot(w, \nabla \phi+D, \nabla \dot{\phi})-w,_{\phi}-D,_{\dot{\phi}}+f_{v \phi}=0 & \forall x \in V \\
(w, \nabla \phi+D, \nabla \dot{\phi}) \cdot n=f_{s \phi} & \forall x \in \partial V
\end{array}
$$

These equations describe the response of the solid from an initial position of state and velocity. The forces $f_{v \phi}$ and $f_{s \phi}$ appears as physical data. In this spirit, the condition $f_{v \phi}=0$ and $f_{s \phi}=0$ has been denoted as the constitutive insulation condition following a terminology due to Polizzotto [18]. The response of a solid under insulation condition has been discussed by several authors, cf. [6], [18], [4], [13].

\section{GENERALIZED STANDARD FORMALISM AND BIOT EQUATION}

In fact, these equations can also be derived in an alternative way, without any assumption on the extended virtual work equation. Indeed, it is established in this section that the governing equations (6) can be also derived directly from the formalism of genneralized standard materials [11]. This formalism states that the dissipative forces, obtained from the expression of the dissipation, are also derived from the dissipation potential. The expression of the dissipation can be obtained in full details from the entropy production of the solid in a thermodynamical analysis, [17], [18]. For the sake of clarity, only a pure mechanical analysis is given here: 


\subsection{Dissipation Analysis}

Indeed, the solid $V$ admits as energy and dissipation potentials:

$$
\mathbf{W}(\mathbf{U})=\int_{V} w(\nabla u, \phi, \nabla \phi) d V, \quad \mathbf{D}(\dot{\mathbf{U}}, \mathbf{U})=\int_{V} D(\dot{\phi}, \nabla \dot{\phi}, \phi) d V
$$

where $\mathbf{U}=(\mathbf{u}, \boldsymbol{\Phi})$ denotes the fields of displacement and internal parameter. Under the applied forces (Bold face uppercase letters as $\Phi$ or $\mathbf{u}$ refer to fields whereas normal letters $\phi$ and $u$ refer to local values)

$$
\mathbf{F} \cdot \delta \mathbf{U}=\int_{V} f_{v u} \cdot \delta u d V+\int_{\partial V} f_{s u} \cdot \delta u d a
$$

and insulation condition, the dissipation of the solid is by definition the unrecoverable part of the received energy per unit time

$$
D_{V}=\mathbf{F} \cdot \dot{\mathbf{U}}-\frac{d}{d t}\left(\mathbf{W}(\mathbf{U})+\mathbf{K}_{\mathbf{t}}\right)
$$

where $\mathbf{K}_{\mathbf{t}}=\int_{V} \rho / 2 \dot{u}^{2} d V$ denotes the kinetic energy. Taking account of the fundamental law of dynamics, it follows that

$$
D_{V}=\int_{V}\left((\sigma-w, \nabla u): \nabla \dot{u}-w,_{\phi} \cdot \dot{\phi}-w, \nabla \phi \cdot \nabla \dot{\phi}\right) d V \geq 0
$$

\subsection{Generalized Standard Formalism}

The dissipation $D_{V}$ is a product of forces and fluxes. For any field of fluxes $(\delta \mathbf{u}, \delta \boldsymbol{\Phi})$ defined on $V$, the power of the dissipative forces $\mathbf{F}_{\mathbf{d}}$ is

$$
\mathbf{F}_{\mathbf{d}} \cdot \delta \mathbf{U}=\int_{V}\left(\left(\sigma-w, \nabla u: \nabla \delta u-w,_{\phi} \cdot \delta \phi-w, \nabla \phi \cdot \nabla \delta \phi\right) d V\right.
$$

The generalized standard formalism consists of admitting that

$$
\mathbf{F}_{\mathbf{d}} \cdot \delta \mathbf{U}=\mathbf{D}, \dot{\mathbf{U}} \cdot \delta \mathbf{U} \quad \forall \delta \mathbf{U}
$$

Thus

$$
\begin{aligned}
& \int_{V}(D, \nabla \dot{u} \cdot \nabla \delta u+(D, \dot{\phi}-\nabla \cdot D, \nabla \dot{\phi}) \cdot \delta \phi) d V+\int_{\partial V} n \cdot D, \nabla \dot{\phi} \cdot \delta \phi d a \\
& =\int_{V}\left((\sigma-w, \nabla u): \nabla \delta u-\left(w,_{\phi} \cdot-\nabla \cdot w, \nabla \phi\right) \cdot \delta \phi\right) d V-\int_{\partial V} n \cdot w, \nabla \phi \cdot \delta \phi d a, \forall \delta u, \delta \phi
\end{aligned}
$$

For any tensor fields $F$ and $G$, the variational condition $\int_{V}(F \cdot \delta \phi+G: \nabla \delta \phi) d V=0$ for all $\delta \phi$, which can be written as $\int_{V}(F-\nabla \cdot G) \cdot \delta \phi d V+\int_{\partial V}(G \cdot n) \cdot \delta \phi d a=0$ for all $\delta \phi$, implies after Haar lemma that $F-\nabla \cdot G=0$ in $V$ and that $G \cdot n=0$ on $\partial V$. It results from a classical argument (Haar lemma in Variational Calculus) that the following equations 
hold:

$$
\begin{aligned}
& \nabla \cdot(\sigma-w, \nabla u-D, \nabla \dot{u})=0 \\
& w,_{\phi}-\nabla \cdot w, \nabla \phi+D, \dot{\phi}-\nabla \cdot D, \nabla \dot{\phi}=0, \quad \forall x \in V \\
& (\sigma-w, \nabla u-D, \nabla \dot{u}) \cdot n=O \\
& (w, \nabla \phi+D, \nabla \dot{\phi}) \cdot n=0, \quad \forall x \in \partial V
\end{aligned}
$$

It is then clear that the governing equations (6) are recovered.

\subsection{Extended Biot Equation}

It follows that the governing equations and associated boundary conditions are the local expressions of a global Biot equation

$$
\mathbf{W}, \mathbf{U}+\mathbf{D}, \dot{\mathbf{U}}=\mathbf{F}
$$

In this spirit, the presence of higher gradients of the internal parameter can also be taken into account. For example, if the expression of the energy includes the second gradient $w(\nabla u, \phi, \nabla \phi, \nabla \nabla \phi)$, the same approach leads to the following bulk equations for $\phi$

$$
w,_{\phi}+D, \dot{\phi}-\nabla \cdot(w, \nabla \phi+D, \nabla \dot{\phi})+\nabla \cdot \nabla \cdot(w, \nabla \nabla \phi+D, \nabla \nabla \dot{\phi})=f_{v} \forall x \in V
$$

and to appropriate boundary conditions.

Finally, for a standard gradient or higher-gradient model, the evolving equations for the displacement and the internal parameter of the solid submitted to a loading path $\mathbf{F}(\mathbf{t})=\left(\mathbf{f}_{\mathbf{v u}}(\mathbf{t}), \mathbf{f}_{\mathbf{s u}}(\mathbf{t})\right)$ are given by an extended expression of Biot equation [2]:

$$
\begin{aligned}
& \frac{\delta w}{\delta u}+\frac{\delta D}{\delta \dot{u}}=f_{v u}-\rho \ddot{u} \\
& \frac{\delta w}{\delta \phi}+\frac{\delta D}{\delta \dot{\phi}}=f_{v \phi} \\
& \forall x \in V \\
& + \text { appropriate boundary conditions }
\end{aligned}
$$

with the popular notation

$$
\frac{\delta w}{\delta \phi}=w,_{\phi}-\nabla \cdot w, \nabla \phi+\nabla \nabla \cdot w, \nabla \nabla \phi-\ldots
$$

\section{ILLUSTRATION IN DAMAGE MECHANICS : DAMAGE LOCALIZATION AND CRACK SIMULATION}

Gradient models have been much considered in visco-plasticity as well as in damage mechanics, cf. [1], [13], [10]. In particular the phase-field method, which is very popular in the study of different phenomena of diffusion and phase change, deals principally with gradient models of visco-elasticity, cf. for example [12]. Many discussions have been devoted to the problem of strain localization and fracture, especially in the numerical computation of elastic-plastic solids. These works deal principally with the insulation case $f_{v \phi}=0$ and $f_{s \phi}=0$ because of the difficulty to define physically these actions. 
The practical interest of gradient models in the modeling of multi-physic phenomena in solids is here considered in the light of the recent works on the problem of strain localization in damage mechanics cf. for example [3], [5], [7], [12].

\subsection{A model of viscous damage}

The internal parameter $\phi \geq 0$ is here the damage indicator, $\phi=0$ if no damage and $\phi=+\infty$ if full damage. A simple visco-plastic model of damage is considered with the following expressions of the energy and of the dissipation potentials

$$
\begin{aligned}
w & =e^{-\alpha \phi} w_{e \ell}(\nabla u)+\frac{h}{2} \phi^{2}+\frac{g}{2}|\nabla \phi|^{2}+w_{c} \phi \\
D & =\frac{\xi}{2 S} \dot{\phi}^{2}+\frac{\eta}{2 S} \nabla \dot{\phi}^{2}
\end{aligned}
$$

where $\alpha, h, g$ and $\xi, \eta$ are constants, $w_{e \ell}$ the elastic energy:

$$
w_{e \ell}=\frac{1}{2} \lambda \epsilon_{k k}^{2}+\mu \epsilon: \epsilon, \epsilon=(\nabla u)_{s}
$$

and $S=S(\nabla u, \phi)$ is a given state-dependent number. From (6), the governing equations for the response of the solid are:

$$
\begin{array}{ll}
\forall x \in V \quad: \quad \nabla \cdot \sigma+f_{v u}=0, \sigma=e^{-\alpha \phi}\left(\lambda \epsilon_{k k} I+2 \mu \epsilon\right) \\
\quad(\xi I-\eta \Delta) \dot{\phi}=S\left(-h \phi+g \Delta \phi+\alpha e^{-\alpha \phi} w_{e \ell}(\nabla u)-w_{c}\right) \\
\forall x \in \partial V: \quad g \phi_{, n}+\frac{\eta}{S} \dot{\phi}_{, n}=0 \\
\forall x \in \partial V_{u}: \quad u=u_{d}(t) \\
\forall x \in \partial V_{f}: \quad \sigma_{n} \cdot n=f_{s u}
\end{array}
$$

It is clear that both gradient terms in the expressions of the energy or dissipation potentials lead to a certain everage operation. The influence of these terms can be discussed separately.

These equations show also in the particular case $S=1$ that an full-equilibrium state for the solid under a given load is a stationary point of the energy functional:

$$
\mathbf{J}(\mathbf{u}, \boldsymbol{\Phi})=\int_{V} w(\nabla u, \phi, \nabla \phi) d V-\int_{V} f_{v u} \cdot u d V-\int_{\partial V} f_{s u} \cdot u d a
$$

since $\delta \mathbf{J}(\mathbf{u}, \boldsymbol{\Phi})=0$ at equilibrium. It has been shown in the works of Bourdin, Francfort and Marigo, cf. [3] that the search for a minimum of this functional is particularly interesting when the internal length scale $\ell$ is introduced as $h=\frac{G}{\ell}, g=G \ell$, cf. [3]. For vanishing $\ell$, the search for full-equilibrium states has a strong connection with the apparition of Griffith cracks of surface energy $G$. This result gives an interesting method to detect the apparition of Griffith cracks as the limit of damage zones, cf. [3], [7], [14].

For $\eta=0$, in order to take into account the fact that the damage could extend only when the pressure is negative i.e. when $\sigma_{k k}>0$, the following choice can be introduced:

$$
S=1 \text { if } \sigma_{k k} \geq 0, \quad 0<S \ll 1 \text { if } \sigma_{k k}<0
$$


The constraint $\dot{\phi} \geq 0$ can also be introduced to describe the irreversible aspect of damage. In this case, the governing equations are

$$
\begin{array}{ll}
\forall x \in V \quad: \quad \nabla \cdot \sigma+f_{v u}=0, \sigma=e^{-\alpha \phi}\left(\lambda \epsilon_{k k} I+2 \mu \epsilon\right) \\
& \xi \dot{\phi}=S<-h \phi+g \Delta \phi+\alpha e^{-\alpha \phi} w_{e \ell}(\nabla u)-w_{c}>^{+} \\
\forall x \in \partial V: \quad & g \phi_{, n}=0 \\
\forall x \in \partial V_{u}: \quad & u=u_{d}(t) \\
\forall x \in \partial V_{f}: \quad & \sigma \cdot n=f_{s u}
\end{array}
$$

where $\mathrm{S}$ is given by $(23)$ and $\langle a\rangle^{+}$is the positive part of $a$ :

$$
<a>^{+}=a \text { if } a>0, \quad<a>^{+}=0 \text { if } a<0 .
$$

\subsection{Numerical simulation}

The system of equations (21) is studied numerically with $\eta=0$ in order to obtained the equilibrium state of the solid under a displacement-controlled loading. A timediscretization by an explicit scheme is adopted

$$
\xi \frac{\phi_{n+1}-\phi_{n}}{t_{n+1}-t_{n}}=\left(S\left(-h \phi+g \Delta \phi+\alpha e^{-\alpha \phi} w_{e \ell}(\nabla u)-w_{c}\right)\right)_{n} .
$$

For $S=1$, it represents exactly the gradient method to obtain a minimum (local) of the energy functional from a given initial state. For different values of $\ell$, the localization of the damage at equilibrium is considered. The Figs. 1, 2, 3 represent three simple examples of crack propagation simulated by damage analysis following the model.

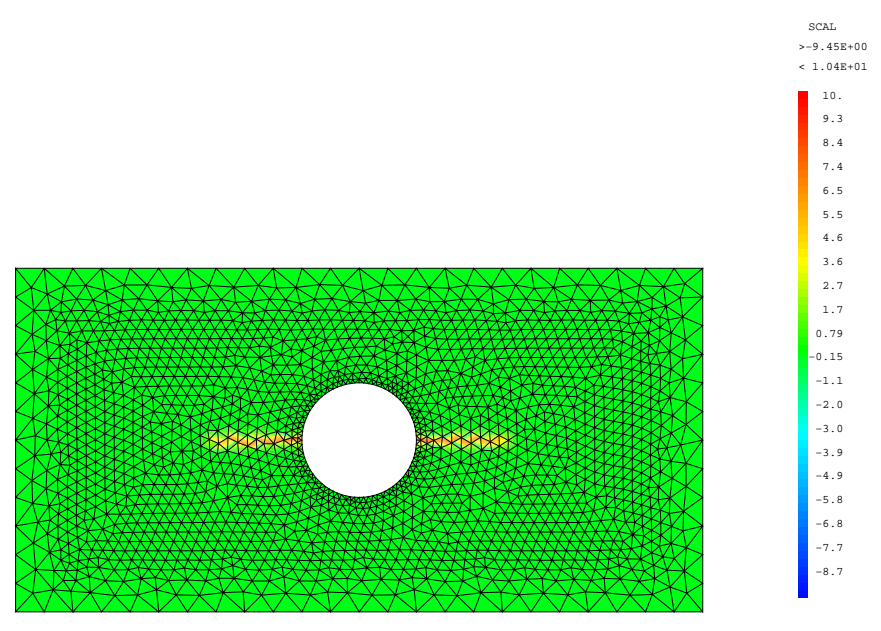

Fig. 1. Damage propagation in a plate with circular hole under displacement control 


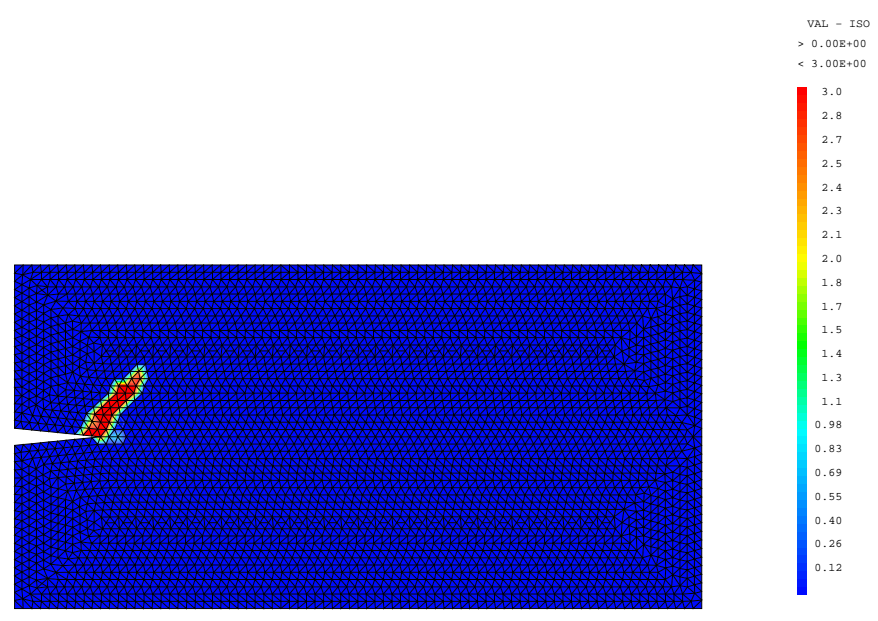

GIBI FECIT

Fig. 2. The propagation in mixed mode simulated by damage

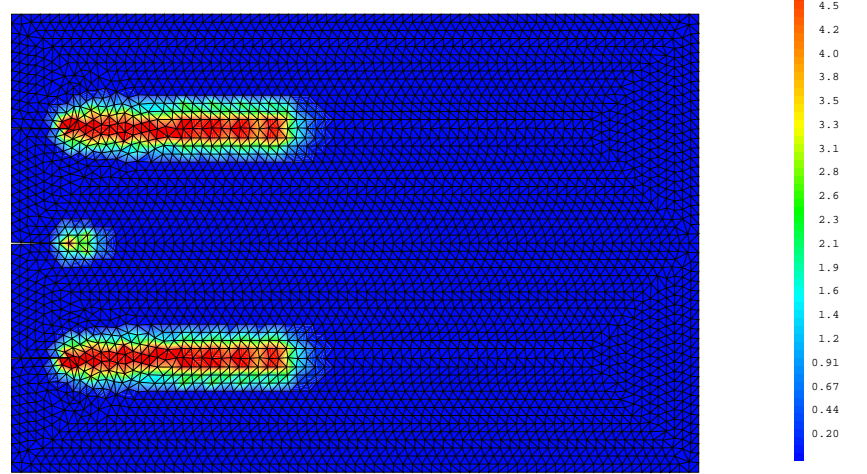

GIBI FECIT

Fig. 3. The propagation and bifurcation of a system of 3 linear cracks

\section{CONCLUSION}

In this paper, the constitutive equations of standard gradient models are conveniently described from the expressions of the energy and the dissipation potentials. Our 
attention is focussed on the derivation of the governing equations as a generalized Biot equation, on the formalism of generalized standard materials and on time-dependent processes such as Visco-elasticity or Visco-Plasticity and Progressive Damage. The interest of gradient terms is explored here in the context of Progressive Damage for the simulation of crack appearance and crack propagation in an elastic solid.

\section{REFERENCES}

[1] E. C. Aifantis, On the microstructural origin of certain inelastic models, J. Eng. Mat. Tech., 106, (1984), 326-330.

[2] M.A. Biot. Mechanics of incremental deformation. Wiley, New York, 1965.

[3] B. Bourdin, Francfort G. A., and Marigo J. J., Numerical experiments in revisited brittle fracture, J. Mech. Phys. Solids, 48, (2000), 797-826.

[4] S. Forest, J. M. Cardona, and R. Sievert, Thermoelasticity of second-grade media, In Maugin, Drouot, and Sidoroff, editors, Continuum Thermodynamics, Kluwer, Dordrecht, (2000).

[5] G. Francfort and J. J. Marigo, Revisiting brittle fracture as an energy minimization problem, J. Mech. Phys. Solids, 46, (1998), 1319-1342.

[6] M. Frémond, Contact unilatéral avec adhérence. une théorie du premier gradient, In G. Del Piero and F. Maceri, editors, Unilateral problems in structural analysis, CISM Course 304, Springer-Verlag, Wien, (1985), 117-137.

[7] M. Frémond, Shape memory alloy: a thermo-mechanical macroscopic theory, In Shape memory alloys, CISM Course 351, Springer-Verlag, Wien, (1996), 3-68.

[8] M. Frémond and B. Nedjar, Damage, gradient of damage and principle of virtual power, Int. J. Solids and Structures, 33, (1996), 1083-1103.

[9] M. E. Gurtin, Generalized ginzburg-landau and cahn-hilliard equations based on a microforce balance, Physica D, 92, (1996), 178-192.

[10] M. E. Gurtin and L. Anand, A theory of strain-gradient plasticity for isotropic, plastically irrotational materials, JMPS, 53, (2005), 1624-1649.

[11] B. Halphen and Q. S. Nguyen, Sur les matériaux standard généralisés, J. Mecanique, 14, (1975), 1-37.

[12] H. Henry and H. Levine, Dynamic instabilities of fracture under biaxial strain using a phasefield model, Phys. Rev. Let., 93:105504, (2004).

[13] E. Lorentz and S. Andrieux, A variational formulation for nonlocal damage models, Int. J. Plasticity, 15, (2003), 119-138.

[14] E. Lorentz and A. Benallal, Gradient constitutives relations: numerical aspect and application to gradient damage, Comp. Meth. Appl. Mech. Eng., 194, (2005), 5191-5220.

[15] Q. S. Nguyen, Variational principles in the theory of gradient plasticity, C. R. Mecanique, 337, (2011), 1-8.

[16] Q. S. Nguyen and S. Andrieux, The non-local generalized standard approach: a consistent gradient theory, C. R. Mecanique, 333, (2005), 139-145.

[17] Q. S. Nguyen, Stability and Nonlinear Solid Mechanics, Wiley, Chichester, (2000).

[18] C. Polizzotto, Unified thermodynamic framework of nonlocal/gradient continuum theories, Eur. J. Mech. A/Solids, 22, (2003), 651-668. 